\title{
Un théorème de la masse positive pour le problème de Yamabe en dimension paire
}

\author{
Pierre Jammes
}

\begin{abstract}
RÉSUmÉ. - Soit $(M, g)$ une variété compacte conformément plate de dimension $n \geq 4$ et de courbure scalaire strictement positive. Selon une théorème de la masse positive dû à Schoen et Yau, le terme constant dans le développement de la fonction de Green du laplacien conforme est strictement positif quand $(M, g)$ n'est pas conforme à la sphère ronde. Sur les variétés spin, Ammann et Humbert en ont donné une démonstration élémentaire, basée sur une preuve de Witten. En utilisant les formes différentielles au lieu des spineurs, nous en donnons une démonstration élémentaire sur les variétés de dimension paire, sans autre hypothèse sur la topologie.

Mots-clefs : théorème de la masse positive, problème de Yamabe, formes différentielles.

AbSTRACT.- Let $(M, g)$ be a compact conformally flat manifold of dimension $n \geq 4$ with positive scalar curvature. According to a positive mass theorem by Schoen and Yau, the constant term in the development of the Green function of the conformal Laplacian is positive if $(M, g)$ is not conformally equivalent to the sphere. On spin manifolds, there is an elementary proof of this fact by Ammann and Humbert, based on a proof of Witten. Using differential forms instead of spinors, we give an elementary proof on even dimensional manifolds, without any other topological assumption.

Keywords : positive mass theorem, Yamabe problem, differential forms.
\end{abstract}

MSC2000 : 58J50

\section{Introduction}

Le problème de Yamabe consiste à trouver dans chaque classe conforme d'une variété compacte une métrique dont la courbure scalaire est constante. Après avoir décelé une erreur dans la solution YYa60 proposée par Yamabe, N.S. Trudinger a pu la corriger dans [Tr68] pour les classes conformes n'admettant pas de métrique à courbure scalaire strictement positive. Les cas restants ont été résolu par T. Aubin Au76 pour les variétés non conformément plate de dimension $n \geq 6$ et par R. Schoen [Sc84] en dimension 3 à 5 et pour les variétés conformément plates (voir [LP87]). 
La démonstration de $\mathrm{R}$. Schoen consiste à se ramener à l'étude d'une variété asymptotiquement euclidienne de la manière suivante : si le laplacien conforme $L_{g}=\frac{4(n-1)}{n-2} \Delta_{g}+\operatorname{scal}_{g}$ est inversible (en particulier s'il existe une metrique de courbure scalaire strictement positive dans la classe conforme de $g$ ), il existe pour tout point $P \in M$ une fonction de Green $\Gamma_{P}$ telle que $L_{g} \Gamma_{P}=\delta_{P}$ au sens des distributions. Comme cette fonction admet un développement de la forme

$$
\Gamma_{P}(x)=\frac{1}{4(n-1) \omega_{n-1} r^{n-2}}+A_{P}+f(x)
$$

où $r=d(P, x), f(x)=O(r)$ et $\omega_{n-1}$ désigne le volume de la sphère canonique $S^{n-1}$, la variété $\left(M \backslash\{P\}, \Gamma_{P}^{\frac{4}{n-2}} g\right)$ est asymptotiquement euclidienne (cf. [LP87]). On appelle souvent projection stéréographique cette construction car elle généralise la projection classique de $S^{n} \backslash\{P\}$ sur $\mathbb{R}^{n}$. Dans [Sc84, $\mathrm{R}$. Schoen montre d'une part qu'on peut résoudre le problème de Yamabe si $A>0$, et d'autre part que la constante $A$ peut s'identifier à la masse de $\left(M \backslash\{P\}, \Gamma_{P}^{\frac{4}{n-2}} g\right)$, la masse étant un invariant riemannien des variétés asymptotiquement plates dont l'étude était initialement motivée par des problèmes issus de la relativité générale.

On est donc ramené au problème de la masse positive, qui consiste à montrer que la masse d'une variété asymptotiquement plate de courbure scalaire positive est positive, l'annulation de la masse caractérisant $\mathbb{R}^{n}$. Il existe différents théorèmes de ce type dans des contextes plus ou moins généraux (voir le survol [He98]). Cette conjecture a d'abord été montrée en dimension $3 \leq n \leq 7$ par Schoen et Yau dans [SY79] et [SY81], et le cas des projections stéréographiques de variétés conformément plates est couvert par [SY88], ce qui achève la résolution du problème de Yamabe.

Par ailleurs E. Witten a proposé dans [Wi81] une démonstration du théorème de la masse positive sur les variétés asymptotiquement euclidiennes utilisant les spineurs (voir aussi [Ba86]). B. Ammann et E. Humbert l'ont simplifiée dans le cas des projections stéréographiques ; sur les variétés conformément plates leur démonstration est particulièrement concise et élégante. Cependant, ces résultats nécessitent l'hypothèse topologique que la variété soit spin. Le but de cet article est de modifier la démonstration de [AH05] de manière à s'affranchir de cette condition. L'argument ne fonctionne qu'en dimension paire, mais il n'y a aucune autre restriction topologique sur la variété, pas même l'orientabilité :

Théorème 1.2 Soit $(M, g)$ une variété riemannienne compacte et conformément plate, de courbure scalaire strictement positive et de dimension $n \geq 4$ 
paire. Pour tout $P \in M$, la masse $A_{P}$ est positive, et $A_{P}=0$ si et seulement si $(M, g)$ est conforme à la sphère canonique.

La démonstration du théorème 1.2 est assez similaire à celle de [AH05]. La principale différence consiste à raisonner sur des formes différentielles au lieu des spineurs, et on utilisera le fait que si $n$ es pair, la formule de Weitzenböck pour les $\frac{n}{2}$-formes est assez simple et bien adaptée au problème.

Je remercie Emmanuel Humbert de m'avoir présenté la démonstration de [AH05], ainsi que Vincent Minerbe de m'avoir recommandé la lecture de [LP87.

\section{Démonstration}

Commençons par quelques rappels techniques concernant le laplacien agissant que les formes différentielles : il est défini par $\Delta=\mathrm{d} \delta+\delta \mathrm{d}$ où la codifférentielle $\delta$ agissant sur les $p$-formes est l'adjoint $L^{2}$ de la différentielle d. Contrairement à une idée répandue, les opérateurs $\delta$ et $\Delta$ sont bien défini quand $M$ n'est pas orientable. Dans la suite, on dira qu'une forme différentielle $\omega$ est harmonique si $\mathrm{d} \omega=\delta \omega=0$ (ce qui est un condition plus forte que $\Delta \omega=0$ ). On peut aussi écrire localement $\delta=(-1)^{n(p+1)+1} * \mathrm{~d} *$ (le signe de la dualité de Hodge $*: \Omega^{p}(M) \rightarrow \Omega^{n-p}(M)$ dépend d'un choix local d'orientation, mais cela n'affecte pas le signe de $\delta$ ), ce qui permet d'appliquer $\delta$ à des formes différentielles qui ne sont pas $L^{2}$. En outre, si la dimension $n$ est paire, l'opérateur $*: \Omega^{\frac{n}{2}}(M) \rightarrow \Omega^{\frac{n}{2}}(M)$ est conformément invariant. En particulier, si $\omega \in \Omega^{\frac{n}{2}}(M)$ alors l'harmonicité de $\omega$ est une propriété conforme (contrairement à $\Delta \omega=0$ ).

Considérons maintenant une variété compacte $\left(M^{n}, g\right)$ de dimension $n$ paire conformément plate et de courbure scalaire positive, et un point $P \in M$. Comme la positivité de $A_{P}$ ne dépend pas du choix de la métrique dans la classe conforme de $g$ ( $\mathrm{Au} 98$, proposition 5.41), on peut en fait supposer que la métrique $g$ est plate dans une petite boule $B_{P}\left(r_{0}\right)$ de rayon $r_{0}$ et centrée en $P$. La première étape de la démonstration consiste à construire une $\frac{n}{2}$-forme harmonique sur $M \backslash\{P\}$, pour ensuite l'utiliser pour estimer la masse de la projection stéréographique de $M$. Cette étape nécessite des outils spécifiques aux formes différentielles.

On commence par construire un modèle euclidien de forme harmonique : soit $\varphi_{0} \in \Lambda^{\frac{n}{2}} \mathbb{R}^{n}$ une forme alternée qu'on identifie à la $\frac{n}{2}$-forme différentielle invariante correspondante sur $\mathbb{R}^{n}$. Cette forme est parallèle, donc $\mathrm{d} \varphi_{0}=\delta \varphi_{0}=0$. L'inversion $i$ par rapport à la sphère unité est un difféomorphisme conforme de $\mathbb{R}^{n} \backslash\{0\}$, on a donc aussi $\mathrm{d} i^{*}\left(\varphi_{0}\right)=\delta i^{*}\left(\varphi_{0}\right)=0$. Si on note $r$ la coordonnée radiale sur $\mathbb{R}^{n}$, on a $i^{*}\left(g_{\text {eucl }}\right)=r^{-4} g_{\text {eucl }}$, donc $\left|i^{*}\left(\varphi_{0}\right)\right|=r^{-n}$ et de 
plus $\nabla_{r}\left(i^{*}\left(r^{n} \varphi_{0}\right)\right)=0$ car la forme $i^{*}\left(r^{n} \varphi_{0}\right)$ est constante le long d'une droite passant par l'origine.

Lemme 2.1 Soit $\varphi_{0} \in \Lambda^{\frac{n}{2}} \mathbb{R}^{n}$. Il existe une $\frac{n}{2}$-forme $\varphi$ harmonique sur $M \backslash\{P\}$ et telle que $\varphi=i^{*}\left(\varphi_{0}\right)+\varphi^{\prime}$ sur $B_{P}\left(r_{0}\right) \backslash P$, $\varphi^{\prime}$ étant une forme lisse sur $B_{P}\left(r_{0}\right)$.

Démonstration : On commence par choisir une fonction cut off $\eta$ qui vaut 1 sur $B_{P}\left(r_{0} / 2\right)$ et 0 en dehors de $B_{P}\left(r_{0}\right)$, et on note $\bar{\varphi}$ la forme définie sur $M \backslash\{P\}$ par $\bar{\varphi}=\eta i^{*}\left(\varphi_{0}\right)$ sur $B_{P}\left(r_{0}\right)$ et prolongée par 0 en dehors. Comme $\mathrm{d} \bar{\varphi}=0$ sur $B_{P}\left(r_{0} / 2\right) \backslash\{P\}$, forme différentielle $\mathrm{d} \bar{\varphi}$ se prolonge de manière lisse en une forme fermée sur $M$ à support dans $B_{P}\left(r_{0}\right)$; le lemme de Poincaré à support compact ([BT82], corollaire 4.7.1) assure alors qu'il existe donc une $\frac{n}{2}$-forme $\theta$ à support dans $B_{P}\left(r_{0}\right)$ telle que $\mathrm{d} \theta=\mathrm{d} \bar{\varphi}$ sur $B_{P}\left(r_{0}\right) \backslash\{P\}$. En particulier, d $\bar{\varphi}$ est la restriction à $M \backslash\{P\}$ d'une forme exacte sur $M$.

Par théorie de Hodge, on peut donc classiquement trouver une forme lisse $\theta_{1}$ telle que $\mathrm{d} \theta_{1}=\mathrm{d} \bar{\varphi}$ sur $M \backslash\{P\}$ et $\delta \theta_{1}=0$ (on utilise ici seulement le fait que $\delta$ est l'adjoint de $\mathrm{d}$, l'hypothèse d'orientation est superflue). Comme on a aussi $\delta \bar{\varphi}=0$ sur $B_{P}\left(r_{0} / 2\right) \backslash\{P\}$, le même argument permet de trouver une forme $\theta_{2}$ lisse et fermée sur $M$ vérifiant $\delta \theta_{2}=\delta \bar{\varphi}$. Il suffit alors de choisir $\varphi=\bar{\varphi}-\theta_{1}-\theta_{2}$.

Remarque 2.2. Dans AH05, la construction d'un spineur harmonique sur $M \backslash\{P\}$ nécessite une condition sur la métrique, à savoir que l'opérateur de Dirac est inversible (par exemple si la courbure scalaire est strictement positive). Ce n'est pas le cas avec les formes différentielles : c'est l'utilisation du lemme de Poincaré qui permet de se dispenser de ce genre d'hypothèse.

Démonstration du théorème 1.2 : Si $\Gamma_{P}$ est la fonction de Green de $L_{g}$ au point $P$, on pose $G=4(n-1) \omega_{n-1} \Gamma_{P}$ et on pose $\tilde{g}=G^{\frac{4}{n-2}} g$. Du fait que $L_{g}(G)=0$, la formule de transformation de la courbure scalaire pour les déformations conforme indique que scal $\tilde{g}=0 \operatorname{sur}(M \backslash\{P\}, \tilde{g})$.

Comme la forme $\varphi$ fournie par le lemme 2.1 est de degré $\frac{n}{2}$, elle reste harmonique sur $(M \backslash\{P\}, \tilde{g})$, et donc $\Delta \varphi=0$. J.-P. Bourguignon a montré ([Bo81, proposition 8.6 et remarque 8.7$)$ que pour les formes de degré $\frac{n}{2}$ en dimension paire, le terme de courbure de la formule de Weitzenböck s'exprime uniquement à l'aide du tenseur de Weyl et de la courbure scalaire (voir aussi [La06], proposition 4.2). Comme $M$ est conformément plate et que scal $\tilde{g}=0$, on a simplement $\Delta \varphi=\nabla^{*} \nabla \varphi=0$ sur $(M \backslash\{P\}, \tilde{g})$. Une intégration par partie donne 
alors

$$
\begin{aligned}
\int_{M \backslash B_{P}(r)}|\nabla \varphi|_{\tilde{g}}^{2} \mathrm{~d} v_{\tilde{g}} & =\int_{M \backslash B_{P}(r)}\left\langle\nabla^{*} \nabla \varphi, \varphi\right\rangle_{\tilde{g}} \mathrm{~d} v_{\tilde{g}}+\int_{S_{P}(r)}\left\langle\nabla_{\nu} \varphi, \varphi\right\rangle_{\tilde{g}} \mathrm{~d} s_{\tilde{g}} \\
& =\int_{S_{P}(r)}\left\langle\nabla_{\nu} \varphi, \varphi\right\rangle_{\tilde{g}} \mathrm{~d} s_{\tilde{g}}=\frac{1}{2} \int_{S_{P}(r)} \partial_{\nu}|\varphi|_{\tilde{g}}^{2} \mathrm{~d} s_{\tilde{g}}
\end{aligned}
$$

où $\nu$ est le vecteur unitaire (pour $\tilde{g}$ ) normal entrant de sphère $S_{P}(r)$ et $\mathrm{d} s_{\tilde{g}}$ la forme volume induite sur $S_{P}(r)$ par $\tilde{g}$.

D'une part, en utilisant le fait que sur les variétés conformément plates la fonction $f$ dans (1.1) peut s'écrire $r \bar{f}(x)$ où $\bar{f}(x)=O(1)$ quand $r \rightarrow 0$ ([LP87, lemme 6.4), on a

$$
\begin{aligned}
|\varphi|_{\tilde{g}}^{2}= & G^{\frac{-2 n}{n-2}}|\varphi|_{g}^{2}=\left(\frac{1}{r^{n-2}}+4(n-1) \omega_{n-1} A+r \bar{f}(x)\right)^{\frac{-2 n}{n-2}}\left|i^{*}\left(\varphi_{0}\right)+\varphi^{\prime}\right|_{g}^{2} \\
= & \left(1+4(n-1) \omega_{n-1} A r^{n-2}+r^{n-1} \bar{f}(x)\right)^{\frac{-2 n}{n-2}}\left|i^{*}\left(r^{n} \varphi_{0}\right)+r^{n} \varphi^{\prime}\right|_{g}^{2} \\
= & \left(1+4(n-1) \omega_{n-1} A r^{n-2}+r^{n-1} \bar{f}(x)\right)^{\frac{-2 n}{n-2}} \times \\
& \left(1+r^{n}\left\langle i^{*}\left(r^{n} \varphi_{0}\right), \varphi^{\prime}\right\rangle_{g}+r^{2 n}\left|\varphi^{\prime}\right|_{g}^{2}\right) .
\end{aligned}
$$

En utilisant le fait que $\nabla_{r}\left(i^{*}\left(r^{n} \varphi_{0}\right)\right)=0$, on en déduit quand $r \rightarrow 0$ que

$$
\frac{\partial}{\partial r}|\varphi|_{\tilde{g}}^{2}=-8 n(n-1) \omega_{n-1} A r^{n-3}+o\left(r^{n-3}\right)
$$

D'autre part, pour $r$ petit, on a aussi $\nu=-G^{-\frac{2}{n-2}} \frac{\partial}{\partial r} \sim-r^{2} \frac{\partial}{\partial r}$ et $\mathrm{d} s_{\tilde{g}}=$ $G^{\frac{2(n-1)}{n-2}} r^{n-1} \mathrm{~d} s \sim r^{-(n-1)} \mathrm{d} s$ où $\mathrm{d} s$ désigne la forme volume canonique de la sphère $S^{n-1}$. En conjonction avec (2.3) et (2.5), on obtient

$$
0 \leq \int_{M \backslash B_{P}(r)}|\nabla \varphi|_{\tilde{g}}^{2} \mathrm{~d} v_{\tilde{g}}=\frac{1}{2} \int_{S_{P}(r)} \partial_{\nu}|\varphi|_{\tilde{g}}^{2} \mathrm{~d} s_{\tilde{g}}=4 n(n-1) \omega_{n-1}^{2} A+o(1)
$$

et on en déduit que $A \geq 0$.

Il reste à traiter le cas où $A=0$. L'inégalité précédente permet d'affirmer que pour toute forme $\varphi_{0} \in \Lambda^{\frac{n}{2}} \mathbb{R}^{n}$, la forme $\varphi$ correspondante sur $(M \backslash\{P\}, \tilde{g})$ est parallèle. On a donc une base de $\frac{n}{2}$-formes différentielles parallèles sur $(M \backslash\{P\}, \tilde{g})$, et on peut en déduire qu'il existe aussi une base de champs de vecteurs parallèles : si on construit un champ de vecteur comme intersection de noyaux de formes parallèles, il sera parallèle. Par conséquent la variété $(M \backslash\{P\}, \tilde{g})$ est plate, donc isométrique à $\mathbb{R}^{n}$, et $(M, g)$ est conforme à la sphère canonique. 


\section{Références}

[AH05] B. Ammann et E. Humbert - « Positive mass theorem for the Yamabe problem on Spin manifolds », Geom. Funct. Anal., 15 (3), p. 567-576, 2005, math.DG/0304043.

[Au76] T. Aubin - « équations différentielles non linéaires et problème de Yamabe concernant la courbure scalaire », J. math. pur. appl., 55, p. 269-296, 1976.

[Au98] T. AubIN - Some nonlinear problems in Riemannian geometry, Springer Verlag, 1998.

[Ba86] R. BARTNIK - « The mass of an asymptotically flat manifold », Commun. Pure Appl. Math., 39, p. 661-693, 1986.

[Bo81] J.-P. Bourguignon - « Les variétés de dimension 4 à signature non nulle dont la courbure est harmonique sont d'Einstein », Invent. Math., 63 (2), p. 263-286, 1981.

[BT82] R. Bотт et L. W. Tu - Differential form in algebraic topology, Springer Verlag, 1982.

[He98] M. Herzlich - « Les théorèmes de masse positive », Sémin. théor. spectr. géom., 16, p. 107-126, 1998.

[La06] M.-L. LABBI - « On Weitzenböck curvature operators », prépublication, 2006, math.DG/0607521.

[LP87] J. LeE et T. PARKer - « The Yamabe problem», Bull. Amer. Math. Soc., 17 (1), p. 37-91, 1987.

[Sc84] R. SCHOEN - « Conformal deformation of a riemannian metric to constant scalar curvature », J. Differential Geom., 20 (2), p. 479-495, 1984.

[SY79] R. Schoen et S.-T. YAU - « On the proof of the positive mass conjecture in general relativity », Comm. Math. Phys., 65 (1), p. 45-76, 1979.

[SY81] R. Schoen et S.-T. YAU - « Proof of the positive mass theorem. II », Comm. Math. Phys., 79 (2), p. 231-260, 1981.

[SY88] R. Schoen et S.-T. YAU - « Conformally flat manifolds, Kleinian groups and scalar curvature », Invent. Math., 92 (1), p. 47-71, 1988.

[Tr68] N.-S. TRUdingeR - « Remarks concerning the conformal deformation of Riemannian structures on compact manifolds », Ann. Sc. Norm. Super. Pisa, 22, p. 265-274, 1968.

[Wi81] E. Witten - « A new proof of the positive energy theorem », Comm. Math. Phys., 80 (3), p. 381-402, 1981.

[Ya60] H. Yamabe - « On a deformation of Riemannian structures on compact manifolds », Osaka Math. J., 12, p. 21-37, 1960.

Pierre JAMMES

Université d'Avignon et des pays de Vaucluse

Laboratoire d'analyse non linéaire et géométrie (EA 2151)

F-84018 Avignon

Pierre.Jammes@univ-avignon.fr 